\title{
Molecular imaging of cell death in vivo by a novel small molecule probe
}

\author{
Revital Aloya • Anat Shirvan · Hagit Grimberg • \\ Ayelet Reshef • Galit Levin • Dvora Kidron • \\ Avi Cohen · Ilan Ziv
}

Published online: 17 October 2006

(C) Springer Science + Business Media, LLC 2006

\begin{abstract}
Apoptosis has a role in many medical disorders, therefore assessment of apoptosis in vivo can be highly useful for diagnosis, follow-up and evaluation of treatment efficacy. ApoSense is a novel technology, comprising low molecularweight probes, specifically designed for imaging of cell death in vivo. In the current study we present targeting and imaging of cell death both in vitro and in vivo, utilizing NST732, a member of the ApoSense family, comprising a fluorophore and a fluorine atom, for both fluorescent and future positron emission tomography (PET) studies using an ${ }^{18} \mathrm{~F}$ label, respectively. In vitro, NST-732 manifested selective and rapid accumulation within various cell types undergoing apoptosis. Its uptake was blocked by caspase inhibition, and occurred from the early stages of the apoptotic process, in parallel to binding of Annexin- $\mathrm{V}$, caspase activation and alterations in mitochondrial membrane potential. In vivo, NST-732 manifested selective uptake into cells undergoing cell-death in several clinically-relevant models in rodents: (i) Cell-death induced in lymphoma by irradiation; (ii) Renal ischemia/reperfusion; (iii) Cerebral stroke. Uptake of NST-
\end{abstract}

Revital Aloya and Anat Shirvan are equal contribution to the paper

R. Aloya - A. Shirvan · H. Grimberg · A. Reshef · G. Levin .

A. Cohen $\cdot$ I. Ziv

NeuroSurvival Technologies (NST) Ltd.,

Petach-Tikva, Israel

\author{
A. Shirvan $(\square)$ \\ NeuroSurvival Technologies (NST) Ltd., \\ 5 Ha'Odem st, P.O. Box 7119, \\ Petach-Tikva 49170, Israel \\ e-mail: Anat@NST.co.il \\ D. Kidron \\ Department of Pathology, Meir Hospital, \\ Kfar-Saba, Israel \\ e-mail: dkidron@clalit.org.il
}

732 was well-correlated with histopathological assessment of cell-death. NST-732 therefore represents a novel class of small-molecule detectors of apoptosis, with potential useful applications in imaging of the cell death process both in vitro and in vivo.

Keywords Apoptosis · Cell death · Imaging · Chemotherapy $\cdot$ ApoSense

\section{Introduction}

Apoptosis is clearly one of the most fundamental biological processes, and in recent years, cumulative data indicate that apoptosis, its activation or dysregulation has a role in almost any medical disorder, either in the etiology or pathogenesis of disease [1-5]. Therefore, molecular imaging of apoptosis can be a highly-useful tool in clinical medical practice. Such imaging can assist in early diagnosis of disease [for example in disorders associated with excessive activation of the process (Alzheimer's disease, Parkinson's disease)], monitoring of disease course (e.g., myocardial infarction, cerebral stroke), or monitoring of response to treatment, such as monitoring of efficacy of anti-tumor agents for cancer, which operate through induction of cell death in the tumor tissue $[6,7]$.

It is therefore highly desirable to have tools for noninvasive imaging of apoptosis in the clinical set-up. Preferably, such tool should be a low-molecular weight probe, amenable for systemic administration in vivo, and capable of performing selective detection of cells undergoing the death process. Preferably, such probe would manifest uptake and intracellular accumulation within the apoptotic cell, in order to allow a high signal/noise ratio. The probe should also be sensitive to detection of the death process from its early stages. Currently, such tool is not available, which prompted us to develop the ApoSense Technology. 
One of the earliest events in apoptosis is alterations in the distribution of phospholipid constituents of the plasma membrane $[8,9]$. Healthy, viable cells manifest asymmetrical distribution of phospholipids in the plasma membrane. Phospholipid scrambling results in exposure of PS on the cell surface, which can be detected by Annexin- $\mathrm{V}$, a $36 \mathrm{kDa}$ protein with high affinity to PS [10-12]. Annexin-V is a highly useful probe in vitro, while its performance in vivo was found to be limited [13], mainly due to pharmacokinetic problems associated with it being a relatively-large protein, with very slow clearance from the blood. In addition, Annexin- $\mathrm{V}$ in vivo manifests a low signal/noise ratio, explained in part, due to its binding only to the cell surface, while not manifesting uptake and intracellular accumulation within the cell in the early stages of apoptosis.

ApoSense is a novel family of low-molecular weight amphipathic apoptosis markers, which target the cell membrane. The compounds do not cross the plasma membrane of an intact viable cell, but perform selective passage through the membrane and accumulation within the cytoplasm of apoptotic cells from the early stages of the death process. Uptake of these compounds is parallel to the characteristic apoptotic features of acquisition of Annexin- $\mathrm{V}$ binding, activation of caspases and loss of mitochondrial membrane potential. Importantly, uptake of the ApoSense compounds through the cell membrane into the apoptotic cell precedes loss of membrane integrity, assessed for example by propidium iodide (PI) exclusion. We have previously reported on this performance of one member of this family, DDC (, $\mathrm{N}^{\prime}$-didansylL-cystine, MW =707) in imaging of cell death in animal models both in vitro and in vivo [14].

Positron emission tomography (PET) with a radio-label such as ${ }^{18} \mathrm{~F}$ is emerging as the leading modality for molecular imaging, enabling non-invasive, sensitive, quantitative and high-resolution imaging of biological processes. To meet the challenge of PET imaging of apoptosis, we therefore developed NST-732 [(5-dimethylamino)-1-napththalenesulfonyl- $\alpha$-ethyl-fluoroalanine; (Fig. 1)], a member of the ApoSense family, having a compact structure $(\mathrm{MW}=368)$ and both a fluorophore (a dansyl group) and a fluorine atom

Fig. 1 Formula of NST-732

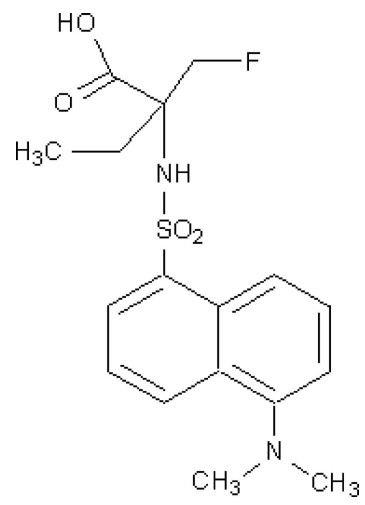

(potentially an ${ }^{18} \mathrm{~F}$ isotope). While the fluorophore may allow fluorescent detection of binding of the compound to the apoptotic cells, ${ }^{18}$ F-labeled NST 732 may allow detection of apoptosis in the clinical set-up, using PET. We now report on the performance of NST-732, as assessed in fluorescence studies, in detection of cell-death in vitro and in vivo, in various cell lines and clinically-relevant animal models.

\section{Materials and methods}

\section{Cell culture}

Human adult T-cell Leukemia Jurkat cells (clone E6-1), mouse lymphoma LY-S cells (L5178y-S) and CT26 colon adenocarcinoma cells (CT26.WT) were obtained from ATCC (Rockville, MD, USA). Cells were cultured in RPMI 1640 medium (Beit Haemek, Israel) supplemented with $2 \mathrm{mM}$ of L-glutamine; $100 \mathrm{units} / \mathrm{ml}$ of penicillin; $100 \mu \mathrm{g} / \mathrm{ml}$ of streptomycine; 12.5 units $/ \mathrm{ml}$ nystatin; $1 \mathrm{mM}$ sodium pyruvate and 10\% FCS. Both Jurkat and LY-S cells were grown in suspension in vertical flasks and seeded at a density of $5 \times 10^{6}$ cells in $10 \mathrm{ml}$ medium. Mouse melanoma cells (B16-F10, ATCC) were maintained in DMEM containing high glucose (4.5 gr/l; Beit Haemek, Israel) supplemented with $4 \mathrm{mM}$ of L-glutamine; 100 units $/ \mathrm{ml}$ of penicillin; $100 \mu \mathrm{g} / \mathrm{ml}$ of streptomycine; $12.5 \mathrm{units} / \mathrm{ml}$ nystatin and $10 \%$ FCS. Cells were cultured in a humidified atmosphere containing $5 \% \mathrm{CO}_{2}$ at $37^{\circ} \mathrm{C}$. Adherent CT26 and B16 cells were grown in flasks and passaged by trypsinization every $2-3$ days (seeded at a density of $1 \times 10^{6}$ cells in $10 \mathrm{ml}$ medium).

\section{Induction of apoptosis in vitro}

Jurkat cells $\left(1 \times 10^{6}\right.$ cells $\left./ \mathrm{ml}\right)$ were treated with $\mathrm{IgM}$ anti-Fas antibody, CH11 (Medical and Biological laboratories, Japan) at a concentration of $0.1 \mu \mathrm{g} / \mathrm{ml}$ for $120-180 \mathrm{~min}$. Cells were harvested and centrifuged at $1600 \mathrm{rpm}$ for $10 \mathrm{~min}$. CT26 and B16 cell lines were incubated for $16 \mathrm{~h}$ with $100 \mu \mathrm{M}$ BiCNU (Bristol-Myers Squibb, Syracuse, NY). Cells were trypsinized, washed with PBS, centrifuged at 1000 for $3 \mathrm{~min}$, and taken for analysis.

\section{Fluorescence microscopy}

Cells $\left(1 \times 10^{6}\right)$ were incubated with NST-732 $(50 \mu \mathrm{M}$ dissolved in NaPPi buffer, pH 7.4) or Annexin-V-FITC (IQ Products, USA). Staining with Annexin-V was according to the manufacture's instructions for $20 \mathrm{~min}$ at RT in a volume of $50 \mu \mathrm{l}$. PI (1:10) (IQ Products, USA) was added immediately before microscopic analysis. Cells were visualized using a fluorescent Olympus microscope (BX51TF; Olympus Optical, U.K.), with UV illumination from a mercury 
lamp, using several fluorescence objectives. NST-732 and PI staining were visualized with excitation at $365 \mathrm{~nm}$ and emission at 420 (band pass) nm, while Annexin-V-FITC was visualized with excitation at $488 \mathrm{~nm}$ and emission at $530 \mathrm{~nm}$.

Fluorescent activated cell sorter (FACS) analysis

Apoptotic cells were detected by flow-cytometry after double staining with either NST-732 and PI or Annexin-V-FITC and PI. Briefly, at the indicated time points following induction of apoptosis by the anti-Fas antibody, $30 \mu 1$ of cell suspension (approximately $1 \times 10^{6} / \mathrm{ml}$ ) were diluted in $300 \mu$ l HEPES buffer (10 mM Hepes and $140 \mathrm{mM} \mathrm{NaCl}, \mathrm{pH}$ 7.4) containing Annexin-V-FITC/PI or $50 \mu \mathrm{M}$ NST-732 and PI. After $20 \mathrm{~min}$ of incubation at room temperature, cells were subjected to analysis using FACS Vantage VE (BD Biosciences, San Jose, CA) and CellQuest software (excitation for NST-732 was at $365 \mathrm{~nm}$ and emission was measured at $530 \mathrm{~nm}$ ). A total of $10^{4}$ events were collected for each sample.

Detection of caspase activation following apoptosis induced by anti-Fas $\mathrm{Ab}$

The methodology was based on the Fluorochrome Inhibitors of Caspase (FLICA), using carboxyfluorescein-labeled fluoromethyl ketone peptide caspase inhibitor (FAM-VADFMK), which produces a green fluorescence (CaspaTag ${ }^{\text {TM }}$ pan-caspase in situ assay kit, Fluorescein, Chemicon, Temecula, CA). FLICA binding specificity was tested by pretreatment with FAM-VAD-FMK according to the manufacturer instructions. Following one-hour incubation with FAMVAD-FMK, cells were washed, stained with PI and analyzed by FACS.

Inhibition of apoptosis with caspase inhibitor

Jurkat cells were pre-incubated with PBS, in the presence of $50 \mathrm{uM} \mathrm{z}$-VAD-FMK in $0.05 \%$ DMSO (Enzyme system products, Dublin, CA) or $0.05 \%$ DMSO for a control test, followed by incubation with $0.1 \mathrm{ug} / \mathrm{ml} \mathrm{CH11}$ anti-Fas antibody. At $180 \mathrm{~min}$, aliquots from each condition were stained with NST732 and PI and analyzed by FACS.

Assessment of mitochondrial membrane potential by TMRE

Assessment of mitochondrial trans-membrane potential was performed using the lipophilic dye tetramethylrhodamine ethyl ester-based (TMRE, Molecular probes, Oregon, USA) [15-16] according to the manufacturer's instructions. TMRE dye displayed a red shift in excitation and emission fluorescence spectra upon delta psi (electric potential across the inner mithochndrial membrane) driven mithochondrial up- take [16]. At the indicated time periods following induction of apoptosis by the anti-Fas Ab, $3 \times 10^{5}$ cells were incubated with $100 \mathrm{nM}$ TMRE at room temperature for $20 \mathrm{~min}$ in HEPES buffer, and the cellular red TMRE fluorescence was detected using FACS (Excitation was at $488 \mathrm{~nm}$ and emission was measured at $580 \mathrm{~nm}$ ).

Renal ischemia and reperfusion injury in rats

Spraque-Dawley rats, weighing 180-250 gr (Harlan laboratories, Jerusalem, Israel) were anesthetized by intraperitoneal injection of the combination of Ketamine, $80 \mathrm{mg} / \mathrm{kg}$ and Xylazine, $10 \mathrm{mg} / \mathrm{kg}$. Renal iscemia was performed according to Liebethal, et al. [17]. After a midline laparatomy incision, the left renal artery was isolated and clamped for $45 \mathrm{~min}$, causing renal ischemia. Reperfusion was then allowed for $24 \mathrm{~h}$. Animals were then injected intravenously with NST$732(35 \mathrm{mg} / \mathrm{kg})$ for a time period of $2 \mathrm{~h}$ and both kidneys were excised, frozen in liquid nitrogen stored at $-80^{\circ} \mathrm{C}$ and further submitted for histological sections.

Experimental cerebral stroke due to middle cerebral artery (MCA) occlusion in mice

Cerebral ischemia was induced in Balb/C mice (10-12 weeks old, Harlan, Jerusalem) by MCA cauterization. Mice were anesthetized, and the temporal bone was exposed. Scraping of the bone up to a minimal hole allowed exposure of the MCA subjecting it to cauterization. After $22 \mathrm{~h}$ from the insult, NST-732 $(70 \mathrm{mg} / \mathrm{kg})$ was injected intravenously, two hours before sacrificing the animals. At $24 \mathrm{~h}$ from induction of the injury, mice were sacrificed by anesthetic overdose, and brains were removed into liquid nitrogen for further histopathology.

Lymphoma model in mice

DBA mice (6-8 weeks old males, Jackson laboratories) were injected subcutaneously with $10^{6}$ LY-S cells in $50 \mu \mathrm{l}$ saline, and were examined daily for tumor formation. For irradiation therapy, mice were irradiated on days 8,9 and 10, once daily, with $6 \mathrm{MV} \mathrm{X}$-rays in Linac apparatus in 3 fractions of 6 Gray/day. Mice received a dose of 1.0 centi-Gray per monitor unit. At day 13 (72 h following the last irradiation) mice were injected intravenously with NST-732 ( $70 \mathrm{mg} / \mathrm{kg}$ ) dissolved as above. Two hours later, tumors were excised, frozen in liquid nitrogen and frozen sections were prepared.

Preparation of histological sections and TUNEL analysis

Four $\mu \mathrm{m}$ thick sections were prepared for microscopic analysis, to follow cells manifesting uptake of NST-732, under 
UV fluorescence objective. Consecutive slides were stained with Hematoxylin and Eosin (H\&E) or with TUNEL (Terminal Deoxynucleotidyltransferase-mediated dUTP Nick End Labeling) [18]. The ApoTag in situ apoptosis detection fluorescent kit (Chemicon International, Temecula, CA) was also used, according to manufacturer's instructions.

Preparation of cytosolic extracts from tumors and quantification of NST 732 binding

Excised tumors were weighted and homogenized (using Heidolph RZR 2020 homogenizer, Heidolph instruments GmbH \& Co.KG, Schwabach, Germany) in cold homogenization buffer containing Tris HCL $50 \mathrm{mM}$ and $0.005 \%$ Triton, $\mathrm{pH}=7.6(1: 7 \mathrm{w} / \mathrm{v})$. Following total homogenization, samples were centrifuged twice at $13,800 \mathrm{rpm}$ for $20 \mathrm{~min}$ at $4{ }^{\circ} \mathrm{C}$ and the supernatants were collected and stored at $-80^{\circ} \mathrm{C}$. For quantification of NST-732 uptake, homogenized tumor samples were aliquoted (in triplicates) onto black Ritter flat bottom micro plates, and read at $360 \mathrm{~nm}$ excitation and $520 \mathrm{~nm}$ emission, using fluorescence micro plate reader (GENious Fl Reader, Tecan, Grodig, Austria). A linear calibration curve was performed using NST-732 at concentrations between $0-25 \mu \mathrm{g} / \mathrm{ml}$. Based on the calibration curve accumulation of NST-732 (expressed as $\mu \mathrm{g} / \mathrm{g}$ tumor tissue) was calculated.

\section{Toxicological studies}

Mice were subjected to an administration of NST-732 in a single dose, up to a dose of $275 \mathrm{mg} / \mathrm{kg}$. In addition, an acute extended single-dose toxicological study was performed in

A

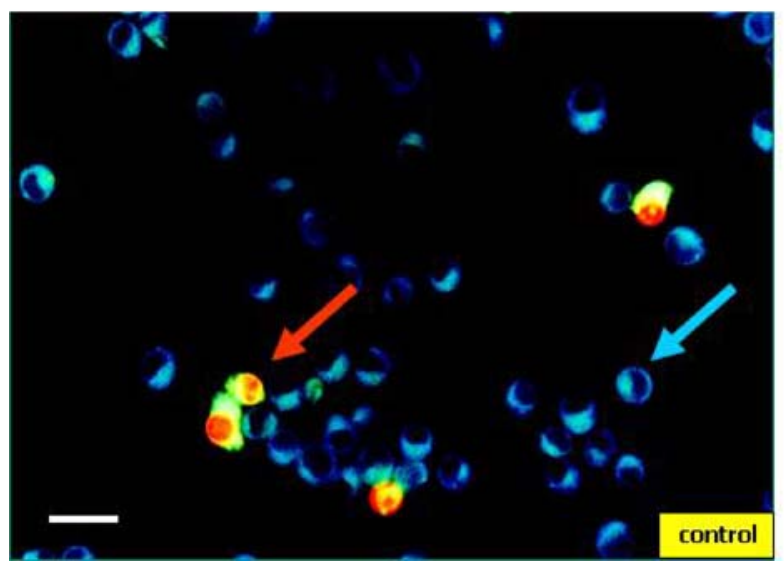

Fig. 2 Detection of Cell death by NST-732 in B16 melanoma cells in vitro; fluorescent microscopy, co-staining with propidium iodide (PI). A. Control cells; most cells are unstained, manifesting only blue auto-fluorescence (blue arrow), un-related to NST-732; few cells manifest binding of both NST-732 and PI (red arrow), reflecting naturallyoccurring cell death in culture. B. Following incubation with BiCNU mice $(n=5)$ at the dose of $5 \mathrm{mg} / \mathrm{kg}$, a dose being more that 2500 -fold higher than the expected human dose for ${ }^{18} \mathrm{~F}$ radiolabled NST-732 for clinical PET studies. Mice were evaluated at $24 \mathrm{~h}, 3$ days and 14 days post dosing for blood hematology and chemistry, and histopathology assessment was performed on day 14 .

\section{Statistical analysis}

Student's $t$-test was used to assess quantitative differences in uptake of NST-732 between control and treated tumors. Statistical significance was defined as $p<0.05$. For qualitative microscopic and flow-cytometric observations, representative results out of at least 3 independent experiments are presented.

\section{Results}

NST732 detects apoptosis in various cell types and in response to various apoptotic triggers

Figure 2 shows fluorescent microscopy of cultured B16 melanoma cells, before and after exposure to BiCNU $(100 \mu \mathrm{M}, 16 \mathrm{~h})$. As shown, the control culture contained very few cells manifesting uptake of NST-732 (green fluorescence), while most of the cells manifested only background cellular blue auto-fluorescence, usually observed in response to UV illumination (Fig. 2A). The cells manifesting uptake of NST-732 reflect cell death, normally occurring in cell cultures. These cells were also stained with PI, thus representing cells in the late stages of cell death, wherein

\section{B}

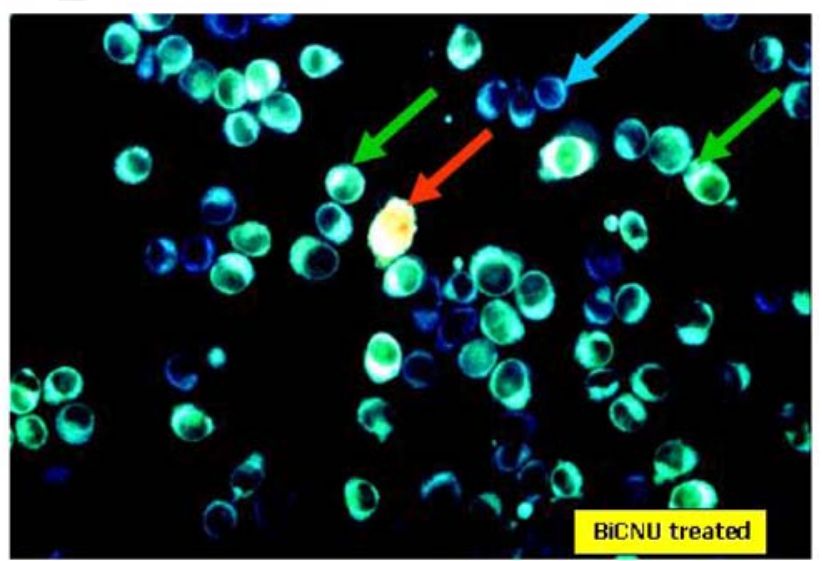

(100 $\mu \mathrm{M}, 16 \mathrm{~h}$ ), numerous cells manifest uptake of NST-732 (green fluorescence). Some of the cells show uptake of NST-732, while excluding PI. These are cells in early apoptosis (EAC; green arrow). Some of the cells show uptake of both NST-732 and PI (red arrow), being cells in the late stages of apoptosis or undergoing a necrotic mode of cell death. Scale bar: $1 \mathrm{~cm}=80 \mu \mathrm{m}$ 


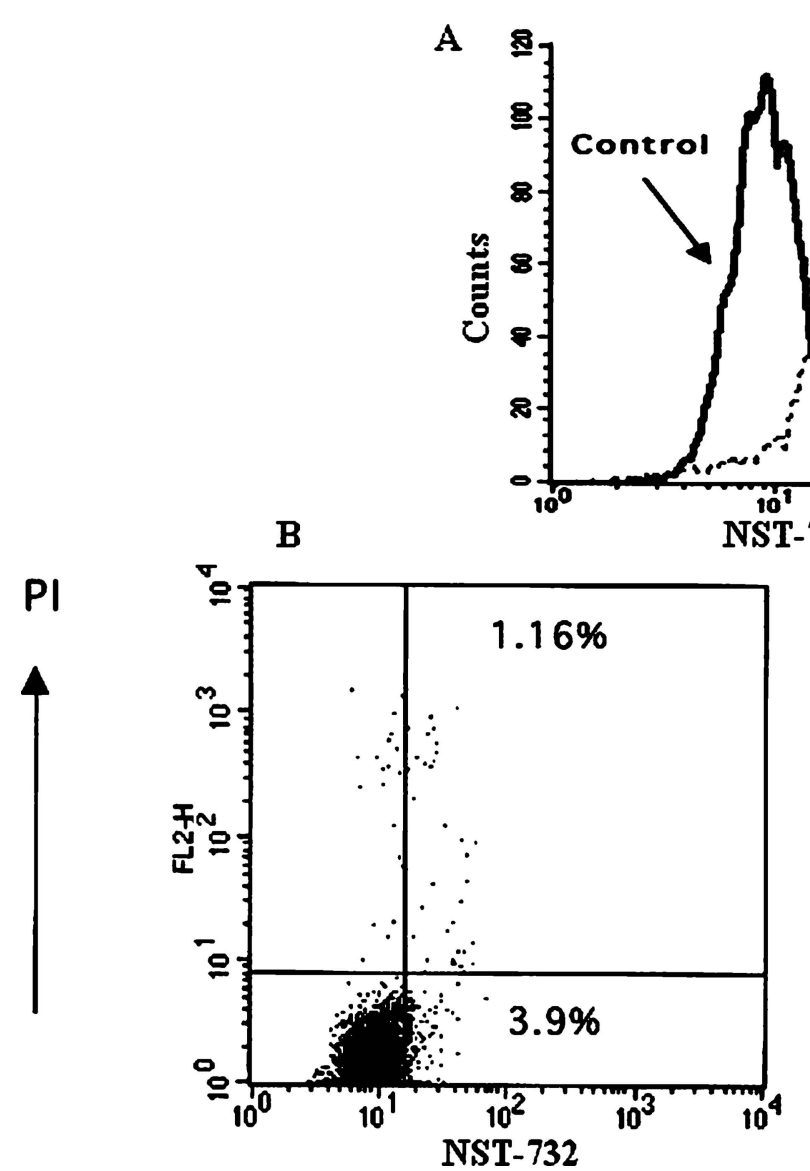

Fig. 3 FACS analysis of uptake of NST-732 by Jurkat cells undergoing apoptosis induced by anti-Fas Ab. A. Control cells (solid line) versus apoptotic cells (dashed line). Analysis of $10^{4}$ cells after incubation with NST-732 $(50 \mu \mathrm{M})$. Apoptosis was associated with a shift of the cell population to a new and distinct peak of higher fluorescence, reflecting enhanced uptake of NST-732 upon induction of apoptosis. B, C. Co-staining of NST-732 versus propidium iodide (PI); control cells (B) versus apoptotic cells $(\mathbf{C})$. As shown, most of the cells undergoing

the membrane loses its integrity, thus enabling ingress of PI. By contrast, upon exposure to the chemotherapeutic drug BiCNU (Fig 2B), numerous cells took up NST-732. Importantly, the compound accumulated within the cytoplasm. Staining of apoptotic cells by NST-732 was stable, and was not reduced by additional washing, suggesting that the uptake is irreversible. Also important is the fact that many of the cells constituting this new population of NST-732-stained cells did not stain with PI. These cells are hereinafter designated Early-Apoptotic Cells (EAC). NST-732 is therefore capable of detecting cells in the early stage of apoptosis, before loss of membrane integrity.

The phenomenon of EAC was constantly observed also with various other cell types and triggers of apoptosis, for example HeLa cells (cervical tumor-derived cells) treated with staurosporine (data not shown), CT26 colon carcinomaderived cells, treated with BiCNU (Fig. 4B), and Jurkat cells induced to undergo apoptosis by treatment with anti-Fas an- apoptosis induced by anti-Fas Ab were in the early stages of the process (EAC), manifesting enhanced uptake of NST-732, while excluding PI (right lower quadrant). This shows the capability of NST-732 to perform selective uptake into apoptotic cells at an early stage, wherein membrane integrity is still maintained. In addition, NST-732 also detected the cells in the late stages of the apoptotic process, manifesting uptake of both NST-732 and PI. The figure describes a representative experiment out of ten performed

tibody (Fig. 3). Detection of EAC by NST 732 was further characterized by FACS. Figure $3 \mathrm{~A}$ shows that treatment with anti-Fas antibody created a shift of the cell population, with formation of a new and distinct peak, characterized by higher fluorescence levels. Dot-plot analysis revealed that the new cell population consists mainly of PI-excluding cells, i.e., cells in early apoptosis (Fig. 3B).

Uptake of NST732 binding is correlated to the activation of the membrane flip-flop mechanism

Sensitivity of the uptake of NST-732 to activation of the flip-flop of membrane constituents occurring in early apoptosis was tested by focusing on EAC, and comparing the time-course of NST-732 uptake by these cells, to the binding of fluorescent-labeled Annexin-V. Annexin-V has high affinity to the headgroups of phosphatidylserine (PS) [10-12]. Since exposure of PS on the cell surface occurs 


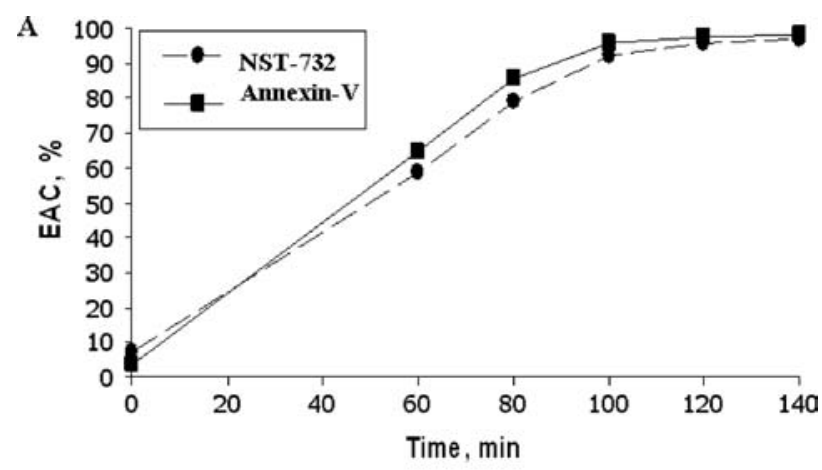

B

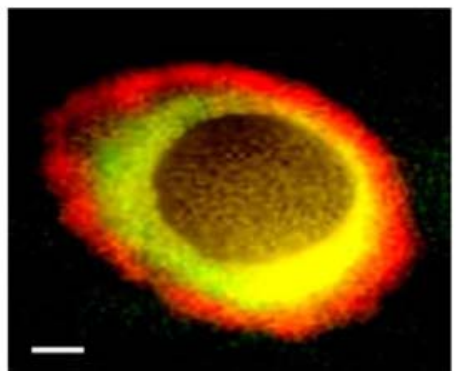

Fig. 4 Detection of early apoptotic events by NST-732: correlation with Annexin-V. A. Time course of binding of NST-732 and Annexin-V to Jurkat cells induced to undergo apoptosis by treatment with anti-Fas Ab. Flow cytometry was performed at different time points after apoptotic induction with anti-Fas Ab. Staining was performed with either NST-732 or annexin-V, versus PI. EAC were identified as cells manifesting NST-732 or Annexin-V uptake while excluding PI. $10^{4}$ cells were counted at each time point. As shown, time-course curves for detection of apoptosis by NST-732 and Annexin-V were practically identical. B. CT26 murine colon carcinoma cell, undergoing apoptosis induced by $\mathrm{BiCNU}$; fluorescent microscopy showing co-staining with NST-732 (green) and Annexin-V Cy3 (red). While Annexin-V localized to the cell membrane, NST-732 manifested accumulation in the cytoplasm. It is noteworthy, that NST-732 was excluded from the nucleus. Scale bar: $1 \mathrm{~cm}=6 \mu \mathrm{m}$

only upon activation of the membrane flip-flop mechanism, binding of exogenously-administered Annexin-V can serve as a reliable reporter on said membrane flip-flop. In the representative experiment described in Fig. 4A, Jurkat cells were induced to undergo apoptosis by treatment with anti-Fas Ab. EAC were detected by flow cytometry as PI-excluding cells. The temporal profile of binding of Annexin- $\mathrm{V}$ or uptake of NST-732 by these EAC after initiation of the apoptotic trigger was co-measured thereafter. As shown, the temporal profile was practically identical. Thus, uptake of NST-732 in early apoptosis, and the apoptotic plasma membrane flip-flop are closely-related. Fig. 4B further demonstrates this observation, showing fluorescent microscopy of a C26 colon carcinoma cell, in the early stages of apoptosis induced by the anticancer agent BiCNU. The cell was co-stained with both fluorophore-labeled Annexin-V (red fluorescence) and NST-732 (green fluorescence). Characteristically, Annexin- $\mathrm{V}$ binds only to the surface of the EAC membrane, where it binds to the exposed PS headgroups. Therefore, it shows a ring-like peripheral staining. By contrast, in parallel to the binding of Annexin-V to the cell surface, NST-732 accumulates within the cytoplasm of the early apoptotic cell.

Uptake of NST-732 is related to caspase activation

Previous studies have shown that interaction through the death receptor CD95 induces apoptosis by formation of a signaling complex at the cell membrane and subsequent caspase- 8 and caspase-3-activation [19]. Therefore, the sensitivity of uptake of NST-732 to the apoptotic process was tested in Jurkat cells, induced to undergo apoptosis by antiFas antibody, but concomitantly treated with the broadspectrum caspase inhibitor Z-VAD-FMK. As shown in a representative experiment in Fig. 5A, addition of the caspase inhibitor resulted in a marked shift of the fluorescent intensity to lower levels, leading to a peak very similar to that of the control cells, suggesting maximal inhibition (Fig. $5 \mathrm{~A}$ ). Thus inhibition of the apoptotic process by a caspase inhibitor blocked uptake of NST-732 by these cells, in spite of their exposure to the pro-apoptotic trigger. Further characterization of these cells by co-staining with PI, revealed that this dramatic effect of caspase inhibition in blocking uptake of NST-732 was indeed on the EAC: In the control culture, the percentage of EAC was 9\%, and upon treatment with the anti-Fas $\mathrm{Ab}$, it rose dramatically to $75 \%$. However, this uptake by the EAC was totally blocked by concomitant treatment with Z-VAD-FMK, to the level of 7.5\% (Fig. 5B).

To further study the relationship between NST-732 uptake by the apoptotic cells and caspase activation, we compared the time-course of uptake of NST-732 into Jurkat cells from initiation of exposure to the apoptotic trigger anti-Fas- $\mathrm{Ab}$, with the respective time-course of uptake of the fluorogenic caspase substrate FAM-VAD-FMK. As shown in Fig. 6A, acquisition of NST-732 uptake by the apoptotic cell was parallel to caspase activation, and at $120 \mathrm{~min}$ exposure to the apoptotic trigger, both NST-732 and the caspase substrate recognized an equal percentage of cells, i.e., at that time point, update of NST-732 matched caspase activation. Interestingly however, at earlier stages of apoptosis induction, NST binding seems to precede detection by the fluorogenic substrate: for example, at sixty minutes, $30 \%$ of the cells already bound NST-732, while only few events were detected by the caspase substrate. This finding suggests that uptake of NST-732 precedes activation of the caspases detectable by FAM-VAD-FMK.

\section{Uptake of NST-732 is correlated with mitochondrial membrane potential disruption}

One of the major events of the apoptotic cascade is disruption of the mitochondrial membrane potential. In order to study the correlation between uptake of NST-732 and changes in 
A

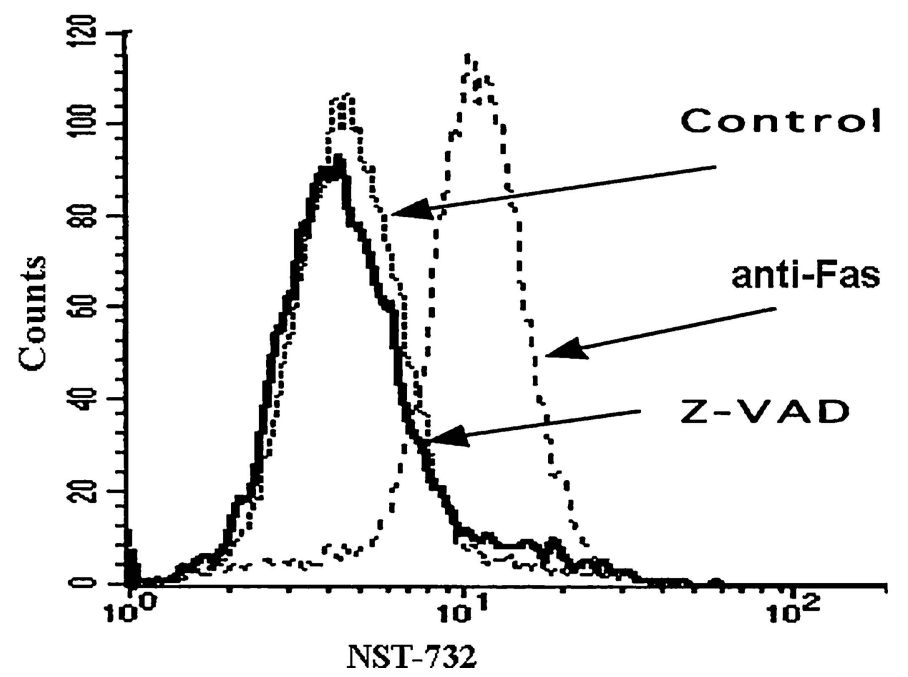

B

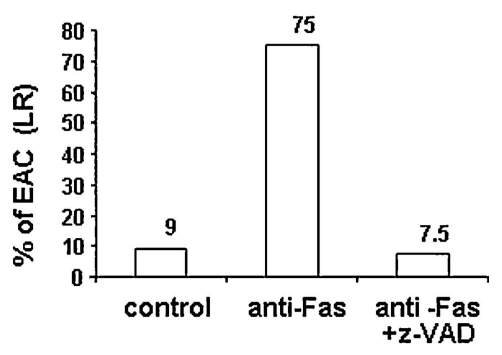

Fig. 5 Inhibition of NST-732 binding by the caspase inhibitor z-VADFMK. Jurkat cells were treated with anti-Fas antibody in the presence or absence of the caspase inhibitor z-VAD $(50 \mu \mathrm{M})$. Following $150 \mathrm{~min}$ of incubation, cells were washed and stained with NST-732 $(50 \mu \mathrm{M})$. PI was added for detection of cells in early apoptosis, and cells were subjected to flow cytometry. A. Uptake of NST-732 by control cells (densely-dotted line) versus cells induced to undergo apoptosis by antiFas Ab, (dotted line), and cells treated with the apoptosis inducer, but in

the mitochondrial membrane potential, Jurkat cells were induced to undergo apoptosis by treatment with Anti-Fas Ab, and the temporal profile of uptake of NST-732 was compared with the signal obtained from tetramethylrhodamine ethyl ester-based (TMRE), a potentiometric fluorescent dye that incorporates into the mitochondria and thus able to measure and report on the mitochondrial membrane potential in a semi-quantitative manner [15, 16]. Exposure to the Anti-Fas $\mathrm{Ab}$ apoptotic trigger led to a progressive decline in mitochondrial membrane potential as reported by TMRE, with a half-life of $75 \mathrm{~min}$. Uptake of NST-732 mirrored this decline in mitochondrial membrane potential (Fig. 6B), and at the time point of $140 \mathrm{~min}$, all cells in culture that showed NST-732 uptake also showed loss of their mithochondrial membrane potential. Interestingly however, acquisition of NST-732 uptake by the cells seemed to precede mitochondrial potential breakdown to some extent: for example, sixty minutes post-treatment with anti-Fas antibody, most of the cells $(91 \%)$ still retained TMRE and only later indicated disruption of mitochondrial membrane potential, while at that time, a large portion of the cells (58.45\%) already indicated their apoptotic destiny by binding NST-732.

NST-732 is a detector of cell death in vivo in various animal models of apoptosis

In order to exemplify the potential utility of NST-732 in detection of cell death in pathological cases, clinically- the presence of the caspase inhibitor (solid line). B. Quantitative analysis of the percentage of cells in early apoptosis (i.e., NST-732-positive, PI-negative cells). As shown, apoptosis was associated with a marked shift of the cell population to a distinct peak of higher fluorescence, reflecting uptake of NST-732 by the apoptotic cells. However, inhibition of the apoptotic process by the caspase inhibitor, practically entirely blocked the uptake of NST-732

relevant animal models were chosen, wherein apoptosis plays a role in the pathogenesis of disease: renal ischemia/reperfusion, and cerebral stroke. In addition, a cancer model, in which detection of cell death may be beneficial for the assessment of tumor response to treatment, was chosen: lymphoma-bearing mice, treated with irradiation. In all these models, NST-732 was administered intravenously, animals were sacrificed thereafter, and the selective uptake of NST-732 only by cells undergoing cell death was demonstrated at the single-cell or whole-organ level, by fluorescent detection. Cell death of tubular cells following renal
ischemia-reperfusion (IR) injury

Unilateral renal ischemia/ reperfusion injury was induced in rats by transient $(45 \mathrm{~min})$ ligation of the renal artery. After reperfusion, NST-732 was administered intravenously as described above. Marked accumulation of NST-732 in the damaged ischemic kidney, but not in the control intact kidney was observed. This was evident at the level of whole-organ imaging (Fig. 7A), showing multiple foci of increased uptake of NST-732, present only in the ischemic kidney, but not in the contralateral kidney. Histological studies by fluorescent microscopy following H\&E and TUNEL staining confirmed that these foci of increased NST732 uptake were indeed foci of cells undergoing cell death (Fig. 7 B-D). 

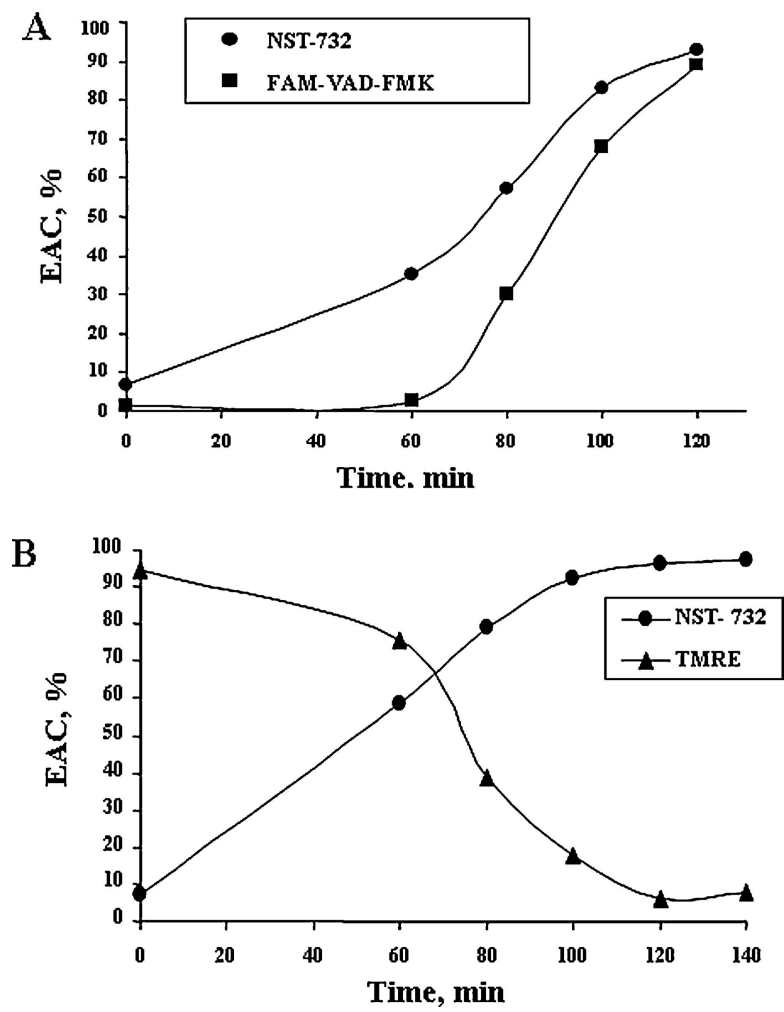

Fig. 6 Correlation of NST-732 uptake with key events in the apoptotic cascade: caspase activation and mitochondrial membrane potential disruption. Jurkat cells were induced to undergo apoptosis by treatment with anti-Fas antibody, and the time-course of NST-732 uptake by the cells was monitored and correlated with key events in the process: A. caspase activation, detected by the caspase substrate FAM-VAD-FMK; B. disruption of mitochondrial membrane potential, detected by the potential-sensitive probe TMRE (see text for details). As shown, NST732 uptake into the apoptotic cells was closely correlated with, and even tended to precede, caspase activation and mitochondrial membrane alterations

\section{Detection of cerebral neuronal cells undergoing cell death} $M C A$ in mice

Cerebral ischemia was induced in mice by unilateral (left) permanent occlusion by cauterization of the MCA. At $24 \mathrm{~h}$ after insult, mice were subjected to an intravenous injection of NST-732. Accumulation of NST-732 in the ischemic area was visualized at the level of whole-organ imaging by stereomicroscope, and is shown in Fig. 8A. Highly selective uptake of NST-732 in the left hemisphere was detected, only in the region supplied by the MCA, while no such uptake was observed in the contralateral hemisphere. Interestingly, the core of the infarct manifested markedly increased uptake, exemplified by a brighter staining (yellow arrow), as compared to the more peripheral regions (red arrowheads). In a microscopic analysis, the region of the infarct showed a high number of individual cells manifesting uptake and intracellular accumulation of NST-732, creating a "starry-sky appearance" (Fig. 8B and C), in clear contrast to the adja- cent intact regions, not affected by the ischemic insult, which did not manifest such uptake. Co-staining with mouse antineuronal nuclei (NeuN) monoclonal antibody confirmed the neuronal identity of the cells (data not shown). TUNEL staining confirmed that the cells manifesting uptake by NST-732 were indeed cells undergoing cell death, showing the characteristic apoptotic DNA fragmentation detectable by TUNEL (Fig. 8D).

Detection by NST-732 of therapy-induced tumor cell death in tumor-bearing mice

Lymphoma-bearing mice were subjected to radiotherapy as described above. NST-732 was then administered intravenously, and two hours later, animals were sacrificed. Tumors from control non-irradiated animals and irradiated animals were subjected to whole-organ fluorescent imaging. As shown in Fig. 9A, the non-irradiated tumor contained only several small foci of NST-732 uptake tumor. However, upon induction of cell death via irradiation, a dramatic increase in NST-732 uptake occurred. Fluorescent microscopy (Fig. 9B) of an area of cells showing high NST-732 uptake, in comparison with Hematoxilin/Eosin $(\mathrm{H} / \mathrm{E})$ staining performed on a consecutive slide (Fig. 9C), indicated that this was indeed an area with disrupted tissue integrity containing numerous cells undergoing cell death, characterized by cytoplasmic acidification and chromatin condensation. TUNEL staining (Fig. 9D) further showed that uptake of NST-732 and positive TUNEL staining were co-localized, confirming that the cells labeled with NST-732 were indeed cells undergoing a death process.

The uptake of NST-732 by the irradiated vs. control tumors was quantified as described above. As shown in Fig. 10, irradiation caused a dramatic increase in uptake of NST-732. While low uptake of NST-732 was observed in the control group $[0.65 \pm 0.2 \mu \mathrm{g} / \mathrm{gr}$ tumor (mean $\pm \mathrm{SEM}$ )], irradiated tumors manifested a 12-fold increase in NST-732 uptake, to a level of $7.93 \pm 1.58 \mu \mathrm{g} / \mathrm{gr}$ tumor $(P<0.001)$.

\section{Toxiclogical studies}

Administration of NST-732, in a single dose, up to a dose of $275 \mathrm{mg} / \mathrm{kg}$ was not associated with any observable adverse effects. The detailed acute single dose toxicological study, performed at a dose of $5 \mathrm{mg} / \mathrm{kg}$ (more than 2500-fold higher than the expected human dose for ${ }^{18} \mathrm{~F}$-radiolabled NST-732 for PET) did not show any significant clinical signs in the blood tests (hematology and chemistry) over a 14-day observation period. The mice had a normal gain in body weight compared to control group, and no abnormality was found in their postmortem histopathological examination. These results indicate a good safety profile for NST-732. 
$A$

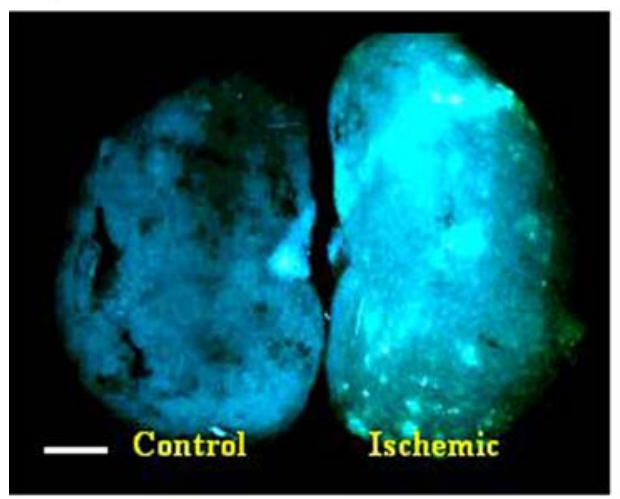

C

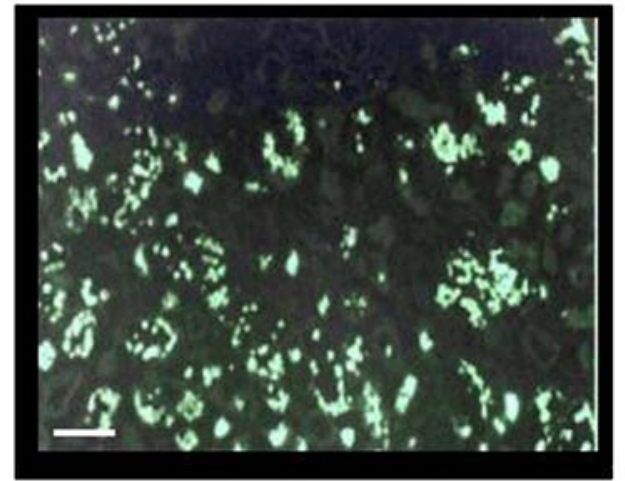

Fig. 7 Fluorescent imaging of apoptosis by systemic administration of NST-732 in vivo following renal ischemia/reperfusion injury. Rats were subjected to unilateral clamping of the renal artery for $45 \mathrm{~min}$, followed by $24 \mathrm{~h}$ of reperfusion. A. Ex-vivo fluorescent whole-organ imaging of the ischemic and the control kidneys. B-D. Histological

\section{Discussion}

The emerging role of apoptosis in almost any medical disorder, either in the etiology or pathogenesis of disease, calls for development of tools for detection of cell death in vivo, in the clinical set-up. While numerous probes for cell death are available in vitro, targeting various stages of the apoptotic process, a barrier currently exists in their use in vivo. Among others, the in vivo situation adds the dimensions of toxicity, immunogenicity, and pharmacokinetics, all leading to the exclusion of practically any probe for apoptosis developed to date. The ApoSense approach was developed to overcome this barrier, by a rationale combining the following considerations: (i) Aiming at a cellular target, which is wellaccepted as an early event of apoptosis. Preferably, this target should be on the cell surface, thus providing easy accessibility of a probe administered systemically in vivo; (ii) Probing this target by a low-molecular weight agent. Such agents, in contrast to large proteins like antibodies or Annexin-V, are characterized by relatively more favorable bio-distribution and clearance, and are more amenable for structural optimization, all important features in construction of a useful

\section{B}

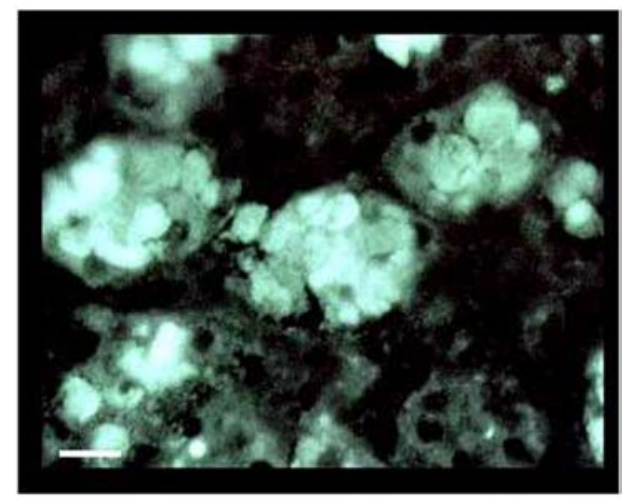

I)

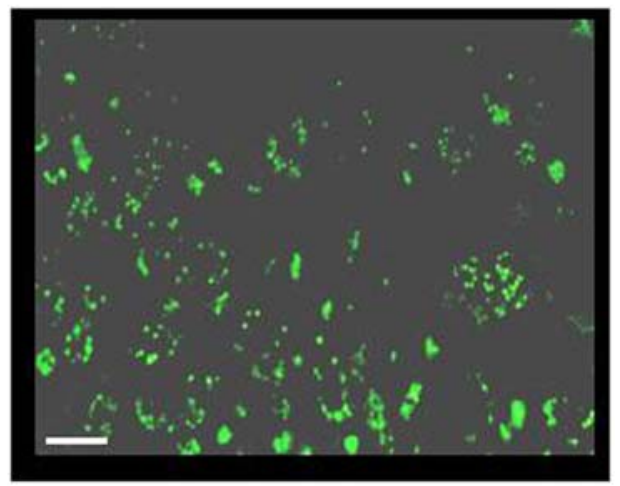

findings in the ischemic kidney: B \& C: accumulation of NST-732 in single tubular cells, (D) verification by TUNEL that cells stained by NST-732 are undergoing cell death. Scale bars: (A) $1 \mathrm{~cm}=125 \mu \mathrm{m}$; (B) $1 \mathrm{~cm}=25 \mu \mathrm{m}$; (C) $1 \mathrm{~cm}=50 \mu \mathrm{m}$

imaging agent; (iii) A probe that will perform intracellular accumulation, thus providing high signal/noise ratio; and (iv) A non-toxic probe suitable for systemic administration.

NST-732 was built in accordance with the above guidelines. It is a novel low molecular weight compound $(\mathrm{MW}=368 \mathrm{Da})$, which manifests selectivity in binding to apoptotic cells, remarkably identical to that of the relatively large protein Annexin-V. As shown in this paper, detection of cell death by NST-732 was found to be universal, i.e., irrespective of cell type or apoptotic trigger, and was found to occur early in the apoptotic process, before disruption of the continuity of the plasma membrane. Uptake of NST-732 is parallel (or perhaps even precedes) caspase activation, and can be completely blocked when the proapoptotic trigger is co-administered with a caspase inhibitor. Time-course of NST-732 uptake is also parallel (or even slightly precedes) the characteristic apoptotic mitochondrial membrane potential disruption. Taken together, NST-732 is an apoptosis-sensitive probe, which uptake accurately corresponds with the induction of the death process or its inhibition, and which also accurately reflects molecular events associated with the apoptotic process. 

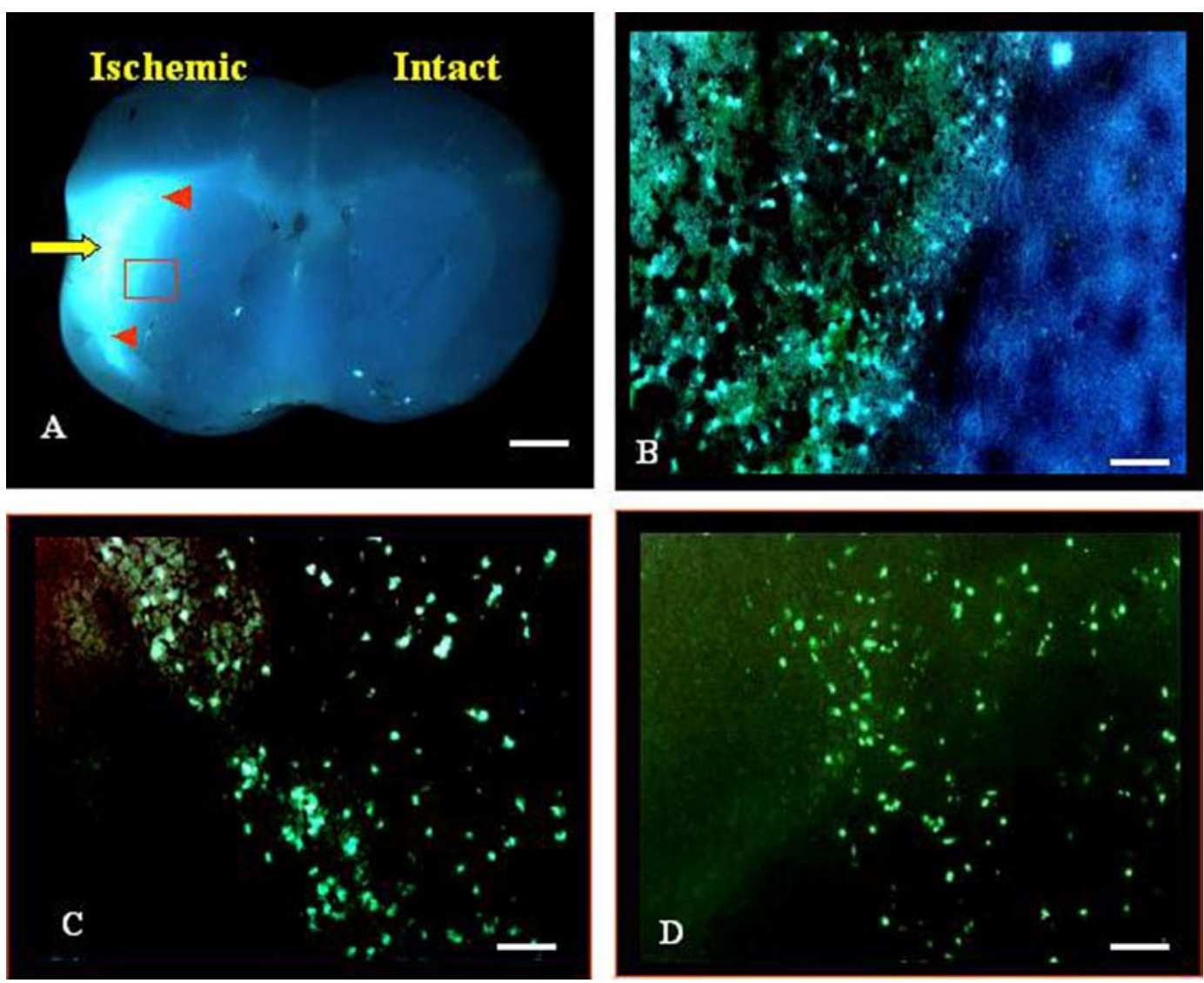

Fig. 8 Fluorescent imaging of apoptosis in acute cerebral stroke by systemic administration of NST-732 in vivo. MCA cauterization was performed in mice as detailed under materials and methods. NST-732 was administered intravenously, and two hours later, animals were sacrificed, and brains were sectioned, subjected to whole-organ imaging and histological assessment. A. Whole-organ imaging, showing intense uptake of NST-732 in the hemispheric region, corresponding with the MCA distribution. Core of the infarct is characterized by more intense signal (marked by yellow arrow), and peripheral damaged areas shows lower intensity of staining (marked by red arrowheads). By contrast,

Both Annexin-V and NST-732 are capable of detecting cells in early apoptosis via membrane associated processes: Annexin- $\mathrm{V}$ by binding to the PS headgroups, and NST-732 by crossing the intact membrane into the cell. Both mechanisms of detection are selective for the death process, as no binding of Annexin-V or uptake of NST-732 are observed in viable cells. However, such mechanism indicates that stages or modes of cell death wherein membrane disruption occurs, such as cells in the late stages of apoptosis, or cells undergoing a necrotic mode of cell death, also manifest uptake of either annexin-V or NST-732. In summary, both Annexin-V and NST-732 are highly specific for cells undergoing cell death, and are excluded from viable cells. Both probes have the unique capability to detect cells in the early stages of apoptosis, in which membrane integrity is maintained, and their binding is parallel to other apoptotic molecular events (e.g, caspase activation, and disruption of

no signal was obtained for other brain regions. (B) Histological analysis revealed that the signal originated from numerous individual cells, manifesting intense intracellular accumulation of NST-732. Shown is an area nourished by the MCA, containing a high density of cell death, taken from the small red frame marked in (A). Note the clear border formed between the intact live tissue and the damaged tissue. (C). An area with a low density of cell death, representing the margins of the injured tissue. (D). TUNEL ex-vivo staining of a consecutive slide as in $\mathrm{C}$, confirmed the identity of these cells as cells undergoing cell death. Scale bars: (A) $1 \mathrm{~cm}=125 \mu \mathrm{m}$; (B) $1 \mathrm{~cm}=50 \mu \mathrm{m}$

mitochondrial membrane potential). Both probes follow the entire apoptotic process, covering its entire scope. Nevertheless, modes or stages of cell death wherein membrane disruption occurs will also be detected by either NST-732 or Annexin-V.

The exact mechanism by which NST-732 targets the cell membrane of the apoptotic cells and crosses it in early apoptosis should be further elucidated. A plausible mechanism which merits further exploration, is a potential association with the dramatic process of scrambling of membrane phospholipids, that occurs early in apoptosis. ApoSense molecules all share amphipathic structures, having specific hydrophobic and charged moieties (see Fig. 1 for NST-732). Extensive structure/function analyses that we performed (to be published separately) have confirmed that integration of all these moieties is required to enable performance of these compounds as detectors of apoptosis. The hydrophobic moiety 


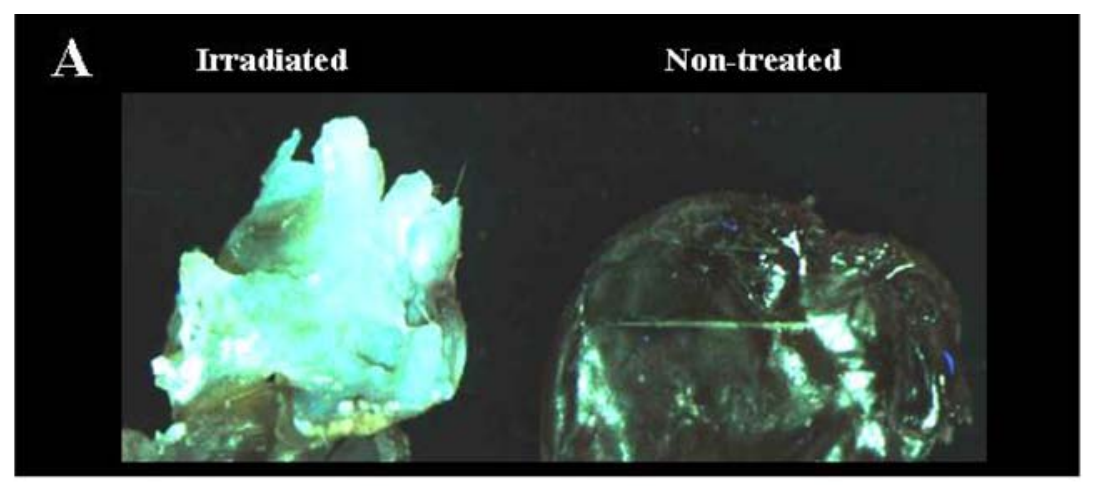

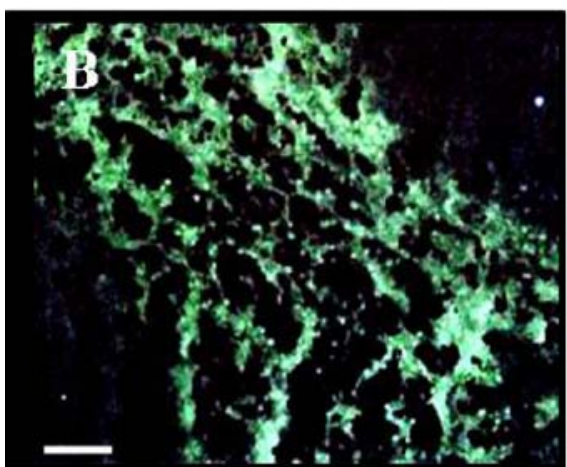

Fig. 9 Uptake of NST-732 in vivo into B16 melanoma cells undergoing cell death. Lymphoma (LY-S) tumors were established in DBA mice. Mice were then treated by irradiation as described in the text. NST-732 was administered intravenously $72 \mathrm{~h}$ after the last dose of irradiation. Two hours later, mice were sacrificed, and tumors were subjected to whole-tumor and microscopic analysis. A. Ex-vivo imaging of the lymphoma control (non-radiated) tumor as compared to irradiated tumor, showing dramatic increase of NST-732 uptake upon irradiation.
B. Fluorescent microscopy of an intense area of cell-death within the irradiated tumor, showing that the signal originated from intracellular accumulation of NST-732 in multiple individual cells undergoing cell death. C. H/E ex-vivo staining of a consecutive slide, showing that the uptake of NST-732 is in high correlation with area of cell death. D. TUNEL ex-vivo staining of a consecutive slide, showing co-localization of the cell-death TUNEL staining with NST-732 uptake, Scale bars: (A) $1 \mathrm{~cm}=300 \mu \mathrm{m}$; (B,C,D) $1 \mathrm{~cm}=100 \mu \mathrm{m}$ seems to provide a membrane anchor, while the charged moiety acts to prohibit crossing the highly hydrophobic membrane core in viable cells. The scrambling process, initiated early in apoptosis and mediated, at least in part, by the scramblase protein(s), substantially reduces this energetic barrier [20]. Consequently, and similar to the respective flip-flop of native phospholipid molecules, the scrambling process may potentially allow flip-flop of NST-732 from the outer to the inner membrane leaflet, and thus its ingress into the cell.

NST-732 manifests several potential advantages over the current state-of-the-art in the field. The main advantage is the intracellular accumulation of the compound, from the early stages of the apoptotic process. This may entail a stronger signal, as compared to Annexin- $\mathrm{V}$, which binds only peripherally, to the external leaflet of the cell membrane. Indeed, a thermodynamic study performed in vitro, wherein Jurkat cells were induced to undergo apoptosis by anti-Fas antibody, revealed that the number of NST molecules accumulated per cell is $1.5 \times 10^{8}$ (not shown). This is about two orders of magnitude higher than the calculated capacity of an apoptotic cell for Annexin-V binding [about $4 \times 10^{6}$ molecules per cell $[21,22]]$. This high number of NST-732 molecules accumulating per apoptotic cell may beneficially contribute to enhancement and amplification of the signal obtained in imaging studies. Another advantage of the compound is that while Annexin-V manifests non-specific binding to the renal cortex, NST-732 does not have this untoward feature. Therefore, NST-732 can be used for detection of renal cell death, as exemplified in the renal ischemia/reperfusion model presented in this study (Fig. 7).

While having a compact structure, NST-732 harbors both a fluorophore, i.e., the dansyl group, and a fluorine atom, potentially being an 18-F isotope, useful for imaging by PET. The dansyl group, via its well-characterized fluorescent properties, allows detection of binding of NST-732 at the single cell level. This allowed for cross-assessment of cellular uptake of the compound versus binding of other markers, for confirmation both in vitro and in vivo as to the identity of the NST-732-binding cells as cells undergoing cell death. It also provided elucidation of the correlation between NST-732 binding and other molecular events in the apoptotic cascade. These fluorescent properties demonstrated the potential utility of NST-732 as detector of cell death in several clinically-relevant animals models in vivo. 


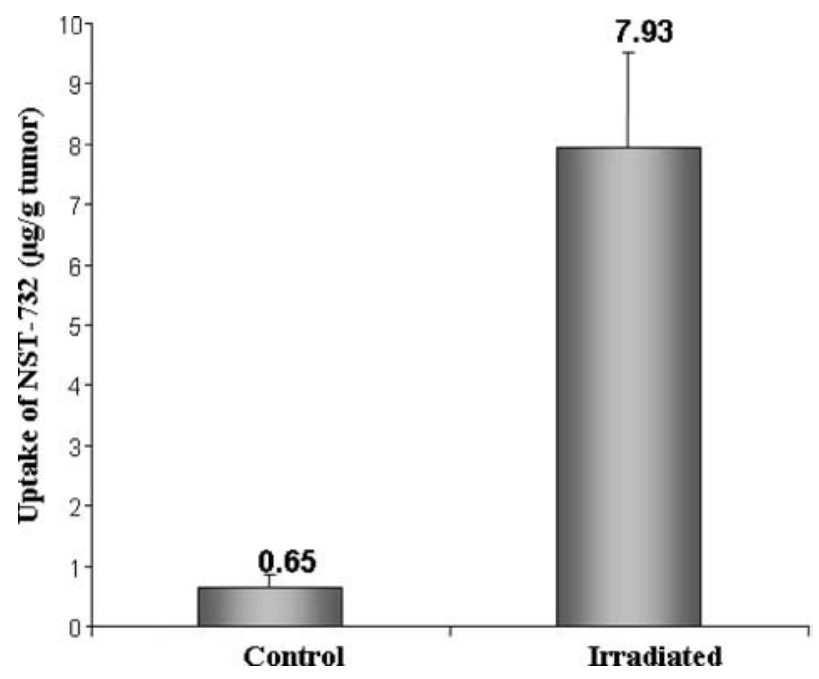

Fig. 10 Uptake of NST-732 in vivo, into lymphoma tumor cells undergoing cell death in response to irradiation. Lymphoma (LY-S) tumors were established in DBA mice. Mice were then treated by irradiation as described in the text. NST-732 was administered intravenously $72 \mathrm{~h}$ after the last dose of irradiation. Two hours later, tumors were harvested from control and irradiated mice, and processed as described under Materials and Methods. Uptake of NST-732 (expressed as $\mu \mathrm{g} / \mathrm{g}$ tumor tissue) was quantified. As shown, average uptake of NST-732 in vivo by the tumors wherein cell death was induced by irradiation $(n=12)$ was 12.5 fold higher than the uptake by the control untreated tumors $(n=8)(p<0.001)$

Based on numerous lines of evidence, it is clear now that apoptosis has a role in almost any medical disorder, either in the etiology or pathogenesis of disease. Detection of cell death in vivo may thus provide better diagnosis, monitoring of disease course or assessment of treatment efficacy in these various disorders. We chose to demonstrate this potential of NST-732 in two major classes of applications: examples of disorders in which apoptosis plays a role in the pathogenesis of disease: renal ischemia/reperfusion, and cerebral stroke; and a scenario wherein detection of cell death may assist in assessment of the efficacy of therapy: monitoring of tumor response to irradiation.

Ischemic or ischemia/reperfusion injury is very prevalent in medical practice, and is a major cause of organ dysfunction and morbidity. Cell death in this clinical situation occurs both in the ischemic and the reperfusion phases. In the model presented in our study, a relatively short-term ischemia (clamping of the renal artery for $45 \mathrm{~min}$ ) was followed by a $24 \mathrm{~h}$ period of reperfusion. Quite amazingly, even such a short ischemic insult was found to be associated with a dramatic process of cell death in the injured kidney. Such process can explain renal failure after systemic hypotension or following kidney transplantation. This load of cell death was wellreported by NST-732, both at the whole-organ level and by histopathology, and confirmed by correlation with TUNEL. Detection of renal cell death by NST-732 may therefore have important implications in early and more accurate diagnosis or organ damage, and monitoring of effect of therapy. In that aspect, ApoSense compounds such as NST-732 are unique, as Annexin-V manifests very high non-specific binding to the renal cortex, thus precluding its use for this purpose.

Cell death is also the major neuropathological substrate in cerebral stroke, and recently, novel therapeutic strategies that emerge for stroke management emphasize the need for detection of the load of stroke-related cell death in order to guide therapy. Thrombolytic therapeutic strategies, e.g., tissue plasminogen activators (tPA), offer a significant potential therapeutic benefit, however with a substantial risk for hemorrhagic complications. This raises an urgent need for assessment of cerebral cell viability following the stroke. In addition, emerging neuro-protective drugs, (e.g., NXY-059, [23]) aiming at inhibiting brain cell death, also require markers for the process, for clinical assessment of their effect on disease course. The study presents the potential usefulness of NST-732 for this purpose. In a murine model of MCA occlusion, systemic administration of NST-732 led to selective accumulation of the compound only in cells undergoing cell death in the region of the infarct, culminating in a strong signal, observed at the level of whole organ imaging.

A major current challenge in oncology is the lack of tools for fast, non-invasive assessment of tumor response to anticancer treatment. The concept of personalized medicine, wherein treatment will be tailored according to the patient's genetic personal makeup, with optimization of therapy in real-time as necessary, still awaits its implementation in clinical oncology. At the level of the individual patient, the lack of such tools is translated into frequent treatment failures, and unnecessary exposure of the patient to the severe adverse effects associated with anti-cancer treatments. At the level of health providers, the current situation leads to suboptimal allocation of therapeutic medical resources. Detection of cell death can provide a direct report on treatment efficacy. The study demonstrates the performance of NST-732 in this context. In lymphoma-bearing mice subjected to irradiation, we found that uptake of NST-732 was markedly increased in response to treatment, respective of its accumulation in the dying cells. Apoptosis is an important mode of cell death induced by anti-cancer treatment, and thus its detection is important for such clinical assessment. However, recent evidence suggests a role also for other modes of cell death in tumor response, such as necrosis, autophagy, mitotic catastrophe or senescence [24, 25]. Moreover, there is often a continuum between features of the different modes of cell death, and various modes of cell death can co-exist in a specific tumor in response to treatment. Importantly, membrane alterations are prominent features of all these modes of cell death, as assessed by Annexin-V and PI studies [24-26]. As NST-732 targets the cell membrane, it is conceivable that it may also be useful for detection of such modes of cell death, extending beyond the classical apoptosis and necrosis 
dichotomy. Obviously however, further research is required to evaluate NST-732 for this purpose.

Taken together, NST-732 administered intravenously in vivo, selectively targeted cells undergoing cell death in various clinically-relevant animal models, thus providing potentially useful information on the extent of cell death associated with the medical disorder or associated with treatment.

Based on these proof-of-concept studies, which support the potential usefulness of NST-732 for imaging cell death in clinical practice, we now proceed towards use of NST732 as a probe for PET. For this purpose we intend to use the fluorine atom inherent in the molecule. Since ${ }^{18} \mathrm{~F}$ is regarded a preferred isotope for PET imaging due to its halflife $(110 \mathrm{~min})$ and the signal that it provides, the synthetic chemistry method to allow ${ }^{18} \mathrm{~F}$ labeling of the compound is now being developed. Such a method should allow rapid and efficient attachment of the isotope in conditions compatible with routine clinical PET radio-chemistry. In view of the possibility of introducing NST-732 as the first smallmolecule probe for clinical PET imaging of apoptosis, we attach great importance to the results of the toxicological studies performed, showing an excellent safety profile of the compound as examined.

\section{References}

1. Thompson CB (1995) Apoptosis in the pathogenesis and treatment of disease. Science 267:1456-1462

2. Rimon G, Bazenet CE, Philpoti KL et al (1997) Increased surface phosphatidylserine is an early marker of neuronal apoptosis. J Neurosci Res 48:563-570

3. Krams SM, Martinez OM (1998) Apoptosis as a mechanism of tissue injury in liver allograft rejection. Semin Liver Dis 18:153-167

4. Olivetti G, Abbi R, Quaini F et al (1997) Apoptosis in the failing human heart. N Eng J Med 336:1131-1141

5. Darzynkiewicz ZJ (1995) Apoptosis in antitumor strategies: modulation of cell cycle or differentiation. J Cell Biochem 58:151-159

6. Wang D, Lippard SJ (2005) Cellular processing of platinum anticancer drugs. Nat Rev Drug Discov 4:307-320

7. Mita M, Tolcher AW (2005) Novel apoptosis inducing agents in cancer therapy. Curr Probl Cancer 29:8-32

8. Savill J (1997) Recognition and phagocytosis of cells undergoing apoptosis. Br Med Bull 53:491-508

9. Martin SJ, Reutelingsperger CP, McGahon AJ et al (1995) Early redistribution of plasma membrane phosphatidylserine is a general feature of apoptosis regardless of the initiating stimulus: inhibition by overexpression of BCL-2 and Abl. J Exp Med 182:1545-1556.
10. Koopman G, Reutelingsperger CP, Kuijten GA et al (1994) Annexin $\mathrm{V}$ for flow cytometric detection of phosphatidylserine expression on B cells undergoing apoptosis. Blood 84:1415-1420

11. Homburg CH, de Haas M, von dem Borne AE et al (1995) Human neutrophils lose their surface Fc gamma RIII and acquire Annexin $\mathrm{V}$ binding sites during apoptosis in vitro. Blood 85:532-540

12. Vermes I, Haanen C, Steffens-Nakken H et al (1995) A novel assay for apoptosis. Flow cytometric detection of phosphatidylserine expression on early apoptotic cells using fluorescein labeled Annexin V. Immunol Methods 184:39-51

13. Vermeersch H, Loose D, Lahorte C et al (2004) 99mTc-HYNIC Annexin-V imaging of primary head and neck carcinoma. Nucl Med Commun 25:259-263

14. Damianovich M, Ziv I, Heyman N et al (2006) Aposense: a novel technology for functional molecular imaging of cell death in models of acute renal tubular necrosis. Eur J Nucl Med Mol Imaging 33(3):281-291

15. Ehrenberg B, Montana V, Wei MD (1988) Membrane potential can be determined in individual cells from the nernstian distribution of cationic dyes. Biophys J 53:785-794

16. Scaduto RC Jr, Grotyohann LW (1999) Measurement of mitochondrial membrane potential using fluorescent rhodamine derivatives. Biophys J 76:469-477

17. Lieberthal W, Levine JS (1996) Mechanisms of apoptosis and its potential role in renal tubular epithelial cell injury. Am J Physiol 271:F477-F488

18. Gavrieli Y, Sherman Y, Ben-Sasson SA (1992) Identification of programmed cell death in situ via specific labeling of nuclear DNA fragmentation. J Cell Biol 119:493-501

19. Hirata H, Takahashi A, Kobayashi S et al (1998) Caspases are activated in a branced protease cascade and control distinct downstream processes in Fas-induced apoptosis. J Exp Med 187:587-600

20. Bevers EM, Comfurious P, Dekkers DW, Zwaal RF (1999) Lipid translocation across the plasma membrane of mammalian cells. Biochim Biophys Acta 1439:317-330

21. Bennett MR, Gibson DF, Schwartz SM et al (1995) Binding and phagocytosis of apoptotic vascular smooth muscle cells is mediated in part by exposure of phosphatidylserine. Circ Res 77:1136-1142

22. Tait JF, Smith C, Wood BL (1999) Measurement of phosphatidylserine exposure in leukocytes and platelets by whole-blood flow cytometry with Annexin V. Blood cells Mol Dis 25:271-278

23. Lees KR, Zivin JA, Ashwood T et al (2006) NXY-059 for acute ischemic stroke. N Engl J Med 354:588-600

24. Brown JM, Attardi LD (2005) The role of apoptosis in cancer development and treatment response. Nature Reviews 5:231-237

25. Corsten MF, Hofstra L, Narula J, Reutelingsperger CPM (2006) Cunting Heads in the War against Cancer: Defining the Role of Annexin A5 Imaging in Cancer Treatment and surveillance. Cancer Res 66:1255-1260

26. Eom YM, Kim MI, Park SS, Goo MJ, Kwon HJ, Sohn S, Kim WH, Yoon G, Choi KS (2005) Two distinct modes of cell death induced by doxorubicin: apoptosis and cell death through mitotic catastrophe accompanied by senescence-like phenotype. Oncogene 24:4765-4777 